\title{
Optoelectronic Performance of Vertical Cavity Surface Emitting AIGaAs/GaAs QW Laser in Non-Conventional Orientation
}

\author{
Sourav Roy \\ Lecturer, Dept. of EEE \\ Jessore University of Science and \\ Technology, Jessore-7408 \\ Bangladesh
}

\author{
S. M. Tasmeeh Ahsan \\ Lecturer, Dept. of EEE \\ European University of Bangladesh, \\ Dhaka-1207 \\ Bangladesh
}

\author{
Sharadindu Gopal Kirtania \\ Lecturer, Dept. of EEE \\ Jessore University of Science and \\ Technology, Jessore-7408 \\ Bangladesh
}

\begin{abstract}
This paper attempts to address a comprehensive in-depth study on the orientation-dependent optoelectronic performance and power-current profile of lattice matched 670nm $\mathrm{Al}_{0.3} \mathrm{Ga}_{0.7} \mathrm{As} / \mathrm{GaAs}$ Single QW Vertical Cavity Surface Emitting Laser (VCSEL) in MATLAB by solving an eightband k.p Hamiltonian using finite difference method at $\Gamma$ point. The analysis is done along conventional (100) as well as non- conventional (110), (111), (113) and (131) crystal orientations. It is seen that there is a substantial correlation of the energy band dispersion profile and peak gain with change of crystal orientation due to change in energy splitting between conduction band to heavy and light hole. Highest optical gain, maximum optical power and minimum threshold current is obtained in (111) crystal orientation. This numerical result demonstrates that (111)-oriented epilayer can be incorporated into the active region of this laser system in order to attain improved performance for ultrahigh speed lightwave communications technology.
\end{abstract}

\section{General Terms}

MATLAB, Energy band dispersion, PI response of QW Laser

\section{Keywords}

Crystal Orientation, Surface emitting laser, AlGaAs, Hamiltonian Matrix, Valence Band, Optical Gain

\section{INTRODUCTION}

Vertical cavity surface emitting lasers (VCSELs) are made by sandwiching light emitting layers between two highly reflective mirrors grown on the principle of distributed Bragg reflectors (DBRs). Narrow beam divergence, low power consumption, high modulation bandwidth, and easy polarization control are major merits of VCSELs over edge emitting lasers [1]. AlGaAs/GaAs VCSEL was first revealed in 1988 and 1990 at Tokyo Institute of Technology [2] and National Taiwan University [3]. This laser system, emitting within $0.63 \sim 0.9 \mu \mathrm{m}$ is a prominent candidate in optical pumping sources, DVD players, optical disc readers and laser pointers. Latest research suggests that $670 \mathrm{~nm}$ laser radiation is a promising modality in endothelial cell (EC) proliferation (key process in tissue repair) and healing of retinal disease which led us to focus on $670 \mathrm{~nm} \mathrm{AlGaAs} \mathrm{surface-emitting}$ laser [4,5].

However, in contrast to edge-emitting lasers, the high reflectance of the output mirror in VCSEL leads to low quantum efficiency and low output power. If the optical gain in the active region is enhanced, the output-mirror reflectance can be reduced with increase in output power [6]. In recent years, it is unleashed that improved optical gain and threshold current of the lasers can be attained by introducing non-(100)oriented quantum well $(\mathrm{QW})$ into the active region as the separation between heavy hole $(\mathrm{HH})$ and light hole $(\mathrm{LH})$ is orientation-dependent; so the density of states and speed of carrier transition from valence band (VB) maxima to conduction band $(\mathrm{CB})$ minima at $\Gamma$-point. So, crystal orientation is a new way in band structure modifications to optimize QW performance [7].

Modern fabrication techniques such as molecular beam epitaxy (MBE) and metal-organic chemical vapor deposition (MOCVD) permits the growth of crystals in nonconventional orientation. Few results on orientation-dependent growth of InGaAs/InP QW are found in literature [8, 9]. Ohtoshi et al. reported the dependence of optical gain on crystal orientation in InGaAs/InP surface emitting laser [6]. In 2012, M.M. Hasan reported crystallographic orientation-dependent optical properties of GaInSb mid-infrared QW laser on GaSb substrate [10]. To best of our knowledge, the optical emission profile and P-I profile of AlGaAs based VCSEL in nonconventional orientation is still unavailable. Hence, to explore the performance of this laser in arbitrary crystal orientations in details, numerical modeling is necessary.

The purpose of this research is to discuss VCSELs that employ non-(100)-oriented lattice matched quantum wells (QWs) and hence to develop a numerical model to study the energy band structure, emission profile and P-I characteristics as a function of different crystal orientations. Here, we calculate for the first time optoelectronic properties related to arbitrary orientations at Brillouin-zone center to estimate performance of $\mathrm{AlGaAs} / \mathrm{GaAs} \mathrm{QW}$ VCSEL. The present analysis is based on $8 * 8$ k.p Hamiltonian which includes spinorbit interaction with heavy and light hole. To investigate the optoelectronic properties in non-(100) orientation, spinor rotations are used to modify the wave vectors and Hamiltonian matrix in conventional crystal orientation.

The description of this paper is designed as follows: the theoretical formula to calculate optical properties of the $\mathrm{AlGaAs} / \mathrm{GaAs} \mathrm{QW}$ are mentioned in section 2. Results and discussions on the energy band structure and optical emission spectra are illustrated in section 3 . Finally, a summary is depicted in section 4 .

\section{THEORY}

\subsection{Laser Structure}

$\mathrm{AlGaAs} / \mathrm{GaAs}$ VCSEL is grown on GaAs substrate. The active region consists of $20 \mathrm{~nm}$ single $\mathrm{Al}_{0.3} \mathrm{Ga}_{0.7} \mathrm{As}$ layer 
sandwiched between $8 \mathrm{~nm}$ GaAs barrier layer and high band gap $\mathrm{Al}_{0.4} \mathrm{Ga}_{0.6} \mathrm{As}$ is used as cladding layer on top and bottom of QW. Two $\mathrm{Al}_{0.12} \mathrm{Ga}_{0.88} \mathrm{As} / \mathrm{Al} \mathrm{As}$ DBRs of mean reflectivity 99.5\% are used as mirrors. In arbitrary crystal orientated laser, the crystal orientation in the active region is (hkl) but all other layers are in (100) crystal orientation. The interface of (100) and (hkl)-oriented layer is joined via wafer bonding technique [11]. Energy Gap $\mathrm{E}_{\mathrm{g}}$ of well is noted as [12]:

$\mathrm{E}_{\mathrm{g}}\left(\mathrm{Al}_{\mathrm{x}} \mathrm{Ga}_{1-\mathrm{x}} \mathrm{As}\right)=\mathrm{E}_{\mathrm{g}}(\mathrm{GaAs})+1.429 * \mathrm{x}-0.14 * \mathrm{x}^{2}$

\section{2 k.p Method of Band-Structure Calculation}

The electronic energy band profile is solved in the envelope approximation using an eight-band $\boldsymbol{k} \bullet \boldsymbol{p}$ Hamiltonian near $\Gamma$ point. The modified Kane $8 \times 8$ Hamiltonian matrix can be given by [13]:

$H_{0} \psi_{h}(z)=E \psi_{h}$

Strain Hamiltonian is neglected since the QW is lattice matched. The components of Hamiltonian matrix $\mathrm{H}_{0}$ are:

$\mathrm{H}_{0}=\left[\begin{array}{cccccccc}A & 0 & V^{*} & 0 & \sqrt{ } 3 V & -\sqrt{2} U & -U & \sqrt{2} V^{*} \\ 0 & A & -\sqrt{ } 2 U & -\sqrt{ } 3 V^{*} & 0 & -V & \sqrt{2} V & U \\ V & -\sqrt{ } 2 U & -P+Q & -S^{*} & R & 0 & \sqrt{\frac{3}{2}} S & -\sqrt{2} Q \\ 0 & -\sqrt{ } 3 & -S & -P-Q & 0 & R & -\sqrt{2} R & \frac{1}{\sqrt{2}} S \\ \sqrt{ } 3 V^{*} & 0 & R^{*} & S & -P+Q & S^{*} & \frac{1}{\sqrt{2}} S^{*} & \sqrt{2} R^{*} \\ -\sqrt{2} U & -V^{*} & 0 & R^{*} & S & -P+Q & \sqrt{2} Q & \sqrt{\frac{3}{2}} S^{*} \\ -U & \sqrt{ } 2 V^{*} & \sqrt{\frac{3}{2}} S^{*} & -\sqrt{2} R^{*} & \frac{1}{\sqrt{2}} S & \sqrt{2} Q & -P-\Delta & 0 \\ \sqrt{2} V & U & -\sqrt{2} Q & \frac{1}{\sqrt{2}} S^{*} & \sqrt{2} R & \sqrt{\frac{3}{2}} S & 0 & -P-\Delta\end{array}\right]$

Here, $\psi_{\mathrm{h}}$ is the conduction and valence band wave function component and $\mathrm{E}$ is the energy of conduction band and three valence bands.

The matrix elements are:

$$
\begin{aligned}
& \mathrm{A}=\mathrm{E}_{\mathrm{c}}-\frac{\hbar^{2}}{2 m_{0}}\left(\mathrm{k}_{\mathrm{x}}{ }^{2}+\mathrm{k}_{\mathrm{y}}{ }^{2}+\mathrm{k}_{\mathrm{z}}{ }^{2}\right) \\
& \mathrm{P}=-\mathrm{E}_{\mathrm{v}}-\gamma_{1} \frac{\hbar^{2}}{2 m_{0}}\left(\mathrm{k}_{\mathrm{x}}{ }^{2}+\mathrm{k}_{\mathrm{y}}{ }^{2}+\mathrm{k}_{\mathrm{z}}{ }^{2}\right) \\
& \mathrm{Q}=-\gamma_{2} \frac{\hbar^{2}}{2 m_{0}}\left(\mathrm{k}_{\mathrm{x}}{ }^{2}+\mathrm{k}_{\mathrm{y}}{ }^{2}+\mathrm{k}_{\mathrm{z}}{ }^{2}\right) \\
& \mathrm{R}=\sqrt{ } 3 \frac{\hbar^{2}}{2 m_{0}}\left[\gamma_{2}\left(\mathrm{k}_{\mathrm{x}}{ }^{2}-\mathrm{k}_{\mathrm{y}}{ }^{2}\right)-2 \mathrm{i} \gamma_{3} \mathrm{k}_{\mathrm{x}} \mathrm{k}_{\mathrm{y}}\right] \\
& \mathrm{S}=-\sqrt{3} \gamma_{3} \frac{\hbar^{2}}{2 m_{0}} \mathrm{k}_{\mathrm{z}}\left(\mathrm{k}_{\mathrm{x}}-\mathrm{ik}_{\mathrm{y}}\right) \\
& \mathrm{U}=\frac{-i}{\sqrt{3}} \mathrm{P}_{0} \mathrm{k}_{\mathrm{z}} \\
& \mathrm{V}=\frac{-i}{\sqrt{6}} \mathrm{P}_{0}\left(\mathrm{k}_{\mathrm{x}}-\mathrm{i} \mathrm{k}_{\mathrm{y}}\right)
\end{aligned}
$$

$\mathrm{P}_{0}$ is the coupling between the conduction and valence bands, $\mathrm{E}_{\mathrm{c}}$ and $\mathrm{E}_{\mathrm{v}}$ are the (unstrained) conduction and valence band energies respectively, $\Delta$ is the spin orbit splitting and $\gamma_{i}$ 's are Luttinger parameters. The energy dispersion profile along (100) orientation is derived as a function of $\mathrm{k}$ for the 8-band k.p theory by diagonalizing the Hamiltonian using numerical finite difference scheme.

\subsection{Euler's Rotation Scheme}

If the active channel is grown on (hkl) crystal orientation, the wave vectors on (100) crystal can be interpreted by the following expression [7]:

$$
\left(\begin{array}{l}
k_{1} \\
k_{2} \\
k_{3}
\end{array}\right)=O_{R}\left(\begin{array}{l}
k_{x} \\
k_{y} \\
k_{z}
\end{array}\right)
$$

The expression of the rotation matrix is as follows [16]:

$$
\begin{aligned}
& O_{R}=\left(\begin{array}{ccc}
\cos \theta \cos \phi & -\sin \phi & \sin \theta \cos \phi \\
\cos \theta \sin \phi & \cos \phi & \sin \theta \sin \phi \\
-\sin \theta & 0 & \cos \theta
\end{array}\right) \\
& \text { Where, } \theta=\tan ^{-1} \frac{\sqrt{\left(h^{2}+k^{2}\right)}}{l} \text { and } \phi=\tan ^{-1} \frac{k}{h}
\end{aligned}
$$

Applying the wave vector in the (100) crystal orientation, the Hamiltonian matrix for (100) crystal orientation can be calculated according to eq. 2 . Then the Hamiltonian matrix in (hkl) orientation can be counted by the simple equation:

$H^{h k l}=U H^{(100)} U^{*}$

Where, $U=R(\theta) R(\phi)$

In this formulae, $R(\theta)$ and $R(\phi)$ are denoted as rotations which transform the (100) orientated valence band Hamiltonian to arbitrary crystal orientated Hamiltonian matrix. The argument of $R(\theta)$ and $R(\phi)$ can be defined by:

$$
\begin{aligned}
R(\theta) & =\left(\begin{array}{cccc}
\alpha^{2} & -\sqrt{3} \alpha^{2} \xi & \sqrt{3} \alpha \xi^{2} & \xi^{3} \\
\sqrt{3} \alpha^{2} \xi & \alpha^{3}-2 \alpha \xi^{2} & -2 \alpha^{2} \xi+\xi^{3} & \sqrt{3} \alpha \xi^{2} \\
\sqrt{3} \alpha \xi^{2} & 2 \alpha^{2} \xi-\xi^{3} & \alpha^{3}-2 \alpha^{2} \xi & -\sqrt{3} \alpha^{2} \xi \\
\xi^{3} & \sqrt{3} \alpha \xi^{2} & \sqrt{3} \alpha^{2} \xi & \alpha^{3}
\end{array}\right) \\
R(\phi) & =\left(\begin{array}{cccc}
e^{i\left(\frac{3}{2}\right) \phi} & 0 & 0 & 0 \\
0 & e^{i\left(\frac{1}{2}\right) \phi} & 0 & 0 \\
0 & 0 & e^{-i\left(\frac{1}{2}\right) \phi} & 0 \\
0 & 0 & 0 & e^{-i\left(\frac{3}{2}\right) \phi}
\end{array}\right)
\end{aligned}
$$

Here, $\alpha=\cos \left(\frac{\theta}{2}\right)$ and $\xi=-\sin \left(\frac{\theta}{2}\right)$

These rotations of the Euler angles $\theta$ and $\phi$ transform the basis states from $(\mathrm{x}, \mathrm{y}, \mathrm{z})$ coordinates to $\left(\mathrm{x}^{\prime}, \mathrm{y}^{\prime}, \mathrm{z}^{\prime}\right)$ coordinates as shown in Fig. 1.

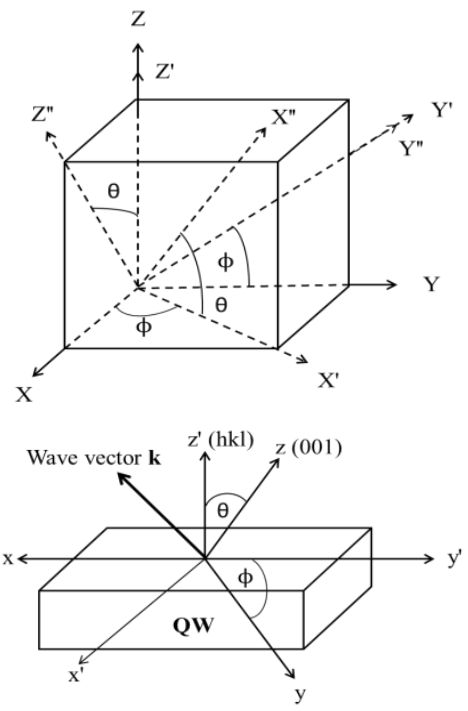

FIG. 1: (a) Spinor Rotation Schematics (b) Configuration of coordinate systems in VCSELs with (hkl)-oriented QWs

\subsection{Optical Gain and Momentum Matrix}

The optical gain as a function of energy for quantum well structure can be approximated by [10]:

$$
\begin{aligned}
& g(E)=\frac{2 q^{2} \hbar}{n \varepsilon_{0} \mathrm{~cm}_{0}^{2} \mathrm{LE}} \\
& \times \sum_{\mathrm{n}, \mathrm{m}} \int_{0}^{\infty} \frac{\frac{\mathrm{k}_{\mathrm{t}} \mathrm{nnm}\left(\mathrm{k}_{\mathrm{t}}\right) \Gamma}{2 \pi}}{\left(\mathrm{E}_{\mathrm{cn}}\left(\mathrm{k}_{\mathrm{t}}\right)-\mathrm{E}_{\mathrm{kpm}}\left(\mathrm{k}_{\mathrm{t}}\right)-\mathrm{E}\right)^{2}+(\Gamma / 2 \pi)^{2}}\left(\mathrm{f}_{\mathrm{c}}{ }^{\mathrm{n}}-\mathrm{f}_{\mathrm{v}}{ }^{\mathrm{m}}\right) \mathrm{d} \mathrm{k}_{\mathrm{t}}
\end{aligned}
$$

Here, $\mathrm{q}$ is denoted as free electron charge, $\hbar$ is the reduced Planck constant, $n$ is the index of refraction, $\mathcal{E}_{0}$ is the free space dielectric constant, c is the speed of light, $\mathrm{E}$ is the photon energy, $E_{c n}$ is the conduction band energy, $E_{k p m}$ is the $\mathrm{m}^{\text {th }}$ valence sub-bands, and $M_{n m}$ is the momentum matrix element in strained quantum well architecture. $\Gamma=\hbar / \tau$ where, $\tau$ is the photon relaxation time. $f_{c}$ and $f_{v}$ are the Fermi levels of conduction and valence bands. 
In a QW structure, the optical momentum matrix element will depend mainly on the transverse electric (TE) polarization of the optical electromagnetic field. The momentum matrix element for TE polarization can be written as [14]:

$$
\begin{aligned}
& \mathrm{M}_{\mathrm{nm}}\left(\mathrm{k}_{\mathrm{t}}\right)[\mathrm{TE}]= \\
& g^{(1)}\left(e^{i\left(\frac{3}{2}\right) \phi}\right)^{*} \cos \theta\left(\frac{m_{0} P_{2}}{\hbar}\right)+g^{(2)}\left(e^{i\left(\frac{1}{2}\right) \phi}\right) \cos \theta\left(\frac{m_{0} P_{2}}{\hbar}\right)- \\
& g^{(3)}\left(e^{i\left(\frac{1}{2}\right) \phi}\right)^{*} \cos \theta\left(\frac{m_{0} P_{1}}{\hbar}\right) \\
& P_{1}^{2}=\frac{\hbar^{2}}{2 \mathrm{~m}_{0}}\left(\frac{m_{0}}{m_{e}^{*}}-1\right) \frac{\left(E_{g}+\Delta_{1}+\Delta_{2}\right)\left(E_{g}+2 \Delta_{2}^{2}\right)}{E_{g}+2 \Delta_{2}} \\
& P_{2}^{2}=\frac{\hbar^{2}}{2 \mathrm{~m}_{0}}\left(\frac{m_{0}}{m_{e}^{*}}-1\right) \frac{E_{g}\left[\left(E_{g}+\Delta_{1}+\Delta_{2}\right)\left(E_{g}+2 \Delta_{2}^{2}\right)\right]}{\left(E_{g}+\Delta_{1}+\Delta_{2}\right)\left(E_{g}+2 \Delta_{2}\right)-\Delta_{1}^{2}}
\end{aligned}
$$

Here, $\Delta_{1}, \Delta_{2}=$ Energy splitting between conduction band to light hole and spin orbit split-off hole

\subsection{P-I Response}

Two-level rate equations that account for carriers in the active and $\mathrm{SCH}$ (Separate Confinement Heterostructure /barrier) layers is converted into equivalent circuit via PSPICE to find output power $\mathrm{P}$ vs. current I response in different crystal orientation $[15,16]$. The model equations found in [15], are

$\frac{d N_{b}}{d t}=\frac{\eta_{i} I}{q V_{\text {barr }}}-R_{b}\left(N_{b}\right)-\frac{N_{b}}{\tau_{\text {capt }}}+\frac{N_{w} V_{a c t}}{V_{\text {barr }}} \frac{N}{\tau_{\text {em }}}$

$\frac{d N}{d t}=\frac{V_{\text {barr }}}{N_{w} V_{\text {act }}} \frac{N_{b}}{\tau_{\text {capt }}}-\frac{N}{\tau_{\text {em }}}-R_{w}(N)-\Gamma_{c} v_{g r} \frac{\alpha(N)}{\phi(S)} S$

$\frac{d S}{d t}=-\frac{S}{\tau_{p}}+N_{w} R_{w \beta}(N)+N_{w} \Gamma_{c} v_{g r} \frac{\alpha(N)}{\phi(S)} S$

$\frac{S}{P_{f}}=\frac{\lambda \tau_{p}}{\eta_{c} V_{a c t} h c}=\vartheta$

$\alpha(N)=G_{0} \ln \left(\frac{R_{w}(N)}{R_{w}\left(N_{0}\right)}\right)$

Gain Saturation $\phi(S)=\frac{1}{1+\varepsilon \Gamma_{C} S}$

$R_{W, b}=A_{W, b} N_{W, b}+B_{W, b} N^{2}{ }_{W, b}+C_{W, b} N^{3}{ }_{W, b}$

Here, Eq. (16) is the rate equation for carrier density $\mathrm{N}_{\mathrm{b}}$ in the $\mathrm{SCH}$ layer and relates its rate of change to the injection current I, the SCH recombination rate, and the carrier exchange between the SCH layers and QW's, namely the rate of carrier capture and emission by the QW's. Eq. (17) accounts for the carrier exchange between the $\mathrm{SCH}$ layers and QW's with capture rate of carriers from the SCH layer and carrier emission from the QW's. Eq. (18) and (19) are for photon density and power. A, B and C are trap aided, radiative and Auger recombination coefficients for well and barrier.

Photon lifetime is calculated: $\tau_{p}^{-1}=v_{g r}\left[\alpha_{i}+\frac{\ln \left(\frac{1}{R_{m}}\right)}{L_{c}}\right]$

Here, $\alpha_{i}=$ internal cavity loss, $\mathrm{R}_{\mathrm{m}}=$ mean reflectivity and $\mathrm{L}_{\mathrm{c}}=$ cavity length

Output power coupling coefficient is determined using: $\eta_{c}=0.5 \ln \left(\frac{1}{R_{m}}\right) /\left[\alpha_{i} L_{c}+\ln \left(\frac{1}{R_{m}}\right)\right.$

Relevant material parameters like lattice constant, deformation potential etc. are taken from [17]. Parameters for evaluating output optical power profile are shown in Table 1.

\section{RESULTS AND DISCUSSION}

Following the k.p method formula given in Section 2.2, the optical properties of lattice matched $20 \mathrm{~nm} \mathrm{ZB} \mathrm{GaAs} /$ $\mathrm{Al}_{0.3} \mathrm{Ga}_{0.7}$ As QW VCSEL structure at the $\Gamma$-point have been studied. The simulation is carried out in MATLAB environment at room temperature. The valence band dispersion curves obtained in (100), (110), (111), (113) and (131) crystal orientations for the first Brillouin zone have been shown in Fig. 2 (a)-(e). To compare the orientation dependent energies, the figures are plotted as a function of wave vector in the same scale. It has been observed, for

values of $\mathrm{k} \neq 0$ (wave vector in the plain direction), the increasing conduction and spin-orbit split off band coupling offer strong anisotropy of the topmost valence band structure at (110) and (113) orientation.

\section{Table 1. Parameters for Output Power Profile}

Name
Current-injection efficiency
Ambipolar Diffusivity

Volume of one QW

Optical confinement factor

Output power coupling coefficient

QW trap-aided recombination coefficient

QW radiative recombination coefficient

QW Auger recombination coefficient

SCH trap-aided recombination coefficient

$\mathrm{SCH}$ radiative recombination coefficient

$\mathrm{SCH}$ Auger recombination coefficient

Phenomenological gain-saturation term

Radiative recombination coupling Volume of SCH

QW capture lifetime

QW emission lifetime

Internal loss of the cavity

Mean mirror reflectivity
Cavity length

$\begin{array}{cc}\begin{array}{c}\text { Symbol } \\ (\text { unit })\end{array} & \text { Value } \\ \eta_{i} & 0.86 \\ D_{a} & 4.4 \\ \left(\mathrm{~cm}^{2} \mathrm{~s}^{-1}\right) & 5 * 10^{-18} \\ V_{a c t}\left(\mathrm{~m}^{3}\right) & 0.022 \\ \Gamma_{c} & 0.522 \\ \eta_{c} & 1.3 * 10^{8} \\ A_{W}\left(\mathrm{~s}^{-1}\right) & \\ B_{W} & 1.4 * 10^{-10} \\ \left(\mathrm{~cm}^{3} \mathrm{~s}^{-1}\right) & \\ C_{W} & 1.3 * 10^{-29} \\ \left(\mathrm{~cm}^{-6} \mathrm{~s}^{-1}\right) & \\ A_{b}\left(\mathrm{~s}^{-1}\right) & 5 * 10^{8} \\ B_{b} & 3.6 * 10^{-11} \\ \left(\mathrm{~cm}^{3} \mathrm{~s}^{-1}\right) & 8 * 10^{-30} \\ C_{b} & \\ \left(\mathrm{~cm}^{-6} \mathrm{~s}^{-1}\right) & 4 * 10^{-17} \\ \varepsilon & 1 * 10^{-4} \\ \beta_{B} & 2.1 * 10^{-16} \\ V_{b a r r}\left(\mathrm{~m}^{3}\right) & 0.97 \\ \tau_{c a p}(\mathrm{ps}) & 10 \\ \tau_{\text {em }}(\mathrm{ps}) & 20 \\ \alpha_{\mathrm{i}}\left(\mathrm{cm}^{-1}\right) & 0.99 \\ \mathrm{R}_{\mathrm{m}} & 100 \\ \mathrm{~L}_{\mathrm{c}}(\mu \mathrm{m}) & \end{array}$

Light emission occurs from lasers due to the allowed transition from conduction band (C) to $\mathrm{HH}$. Other transitions such as intra-subbands transitions are prohibited because it decreases the gain of the laser. Among the intra-subbands, transition probability is highest between $\mathrm{HH}$ and $\mathrm{LH}$, which is minimized when the energy separation between them is higher. For (111) orientation, larger energy spacing between $\mathrm{HH}$ and $\mathrm{LH}$ has been observed which changes the topmost curvature of valence sub-bands and results in the variation of electron and hole mass $\mathrm{m}^{*}$. In order to explain the wavelength dependence of emission profile, the energy separation between the $\mathrm{C}-\mathrm{HH}$ and $\mathrm{HH}-\mathrm{LH}$ bands is estimated from the energy band dispersion profiles and shown in Table 2 . It is found that the energy separation between conduction band minima and valence band maxima changes with crystal plane orientations. It can be noted from this table that the intraband mixing effect is minimum for this laser along (111) orientation. The orientation dependent peak gain can be revealed by the momentum matrix elements that depend on the overlapping wave functions of electrons and holes. It is observed from fig. 3 that transition probability is maximum in (111) orientation and least in (110) and (100) orientations. The lowest band gap and minimum intraband interaction is responsible for the improved result along (111). Here we have calculated the optical gain spectra in different crystal orientations using Eq. (14) and shown in Fig. 4. Here highest gain of $4250 \mathrm{~cm}^{-1}$ is observed in (111) crystal orientation and lowest gain of $2940 \mathrm{~cm}^{-1}$ is in (100) orientation. Highest gain in (111) crystal orientation is obtained due to the small band 
mixing effect and greater transition probability. For this similar issue, minimum gain is observed in (100) crystal orientation. The peak emission wavelength is found to be shifted from 670 to $695 \mathrm{~nm}$.

The crystal orientation-dependent differential gain is calculated and shown in fig.5. Maximum differential gain is obtained along (111) crystal orientation when the injection carrier density is $3.6 * 10^{16} \mathrm{~cm}^{-3}$. Peak values of gain profile from fig. 4 and injection carrier density from fig. 5 are used in PSPICE equivalent circuit to find P-I response.
Optical power-injection current characteristics determined for GaAs/ $\mathrm{Al}_{0.3} \mathrm{Ga}_{0.7} \mathrm{As} \mathrm{QW}$ laser in different crystal orientations are shown in fig. 6. It is found that the maximum optical power of $4.35 \mathrm{~mW}$ and minimum threshold current of $4.96 \mathrm{~mA}$ are obtained in (111) orientated VCSEL structure. On the other hand, the maximum threshold current is evaluated to be $7.6 \mathrm{~mA}$ in (100) crystal orientation.

So, it is quite visible from the above discussion that (111)-oriented vertical cavity surface emitting AlGaAs/GaAs Laser system leads to have improved performance than (100)oriented structure.

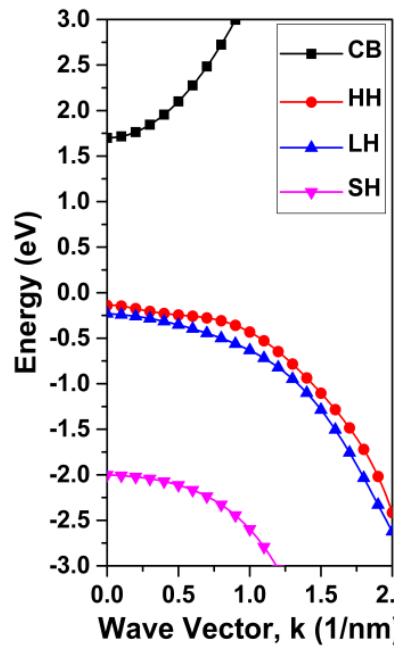

(a)

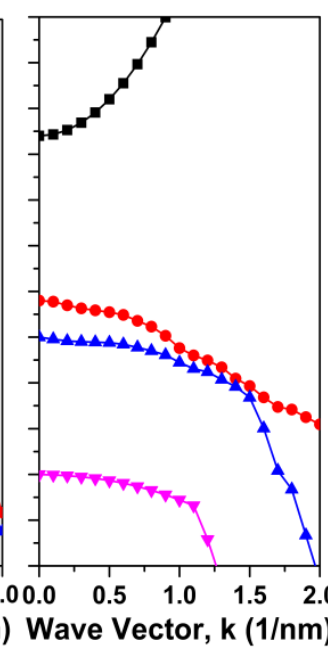

(b)

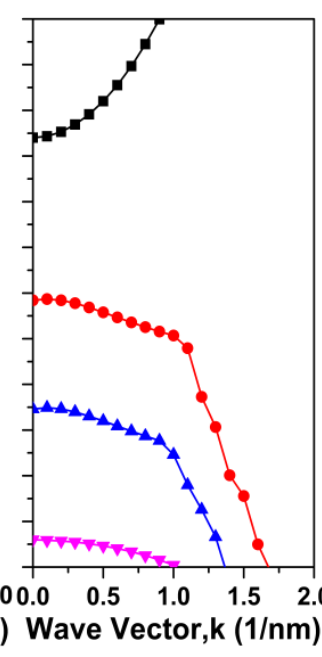

(c)

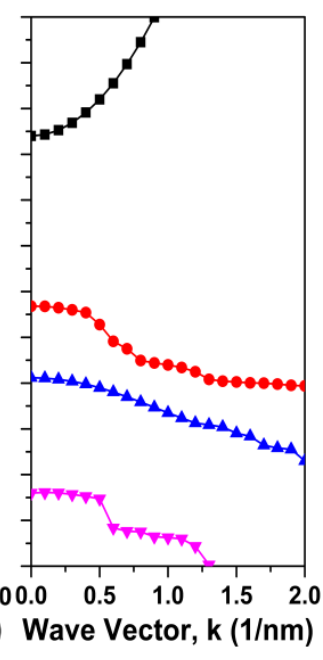

(d)

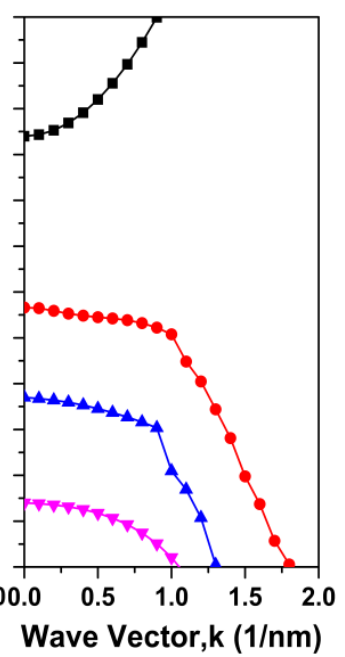

(e)

Fig 2: Energy band dispersion profile of (a) (100) (b) (110) (c) (111) (d) (113) and (e) (131)-oriented AlGaAs/GaAs VCSE Laser [CB=conduction band, HH=Heavy Hole, LH=light hole, $\mathrm{SH}=$ spin-orbit split-off hole]

Table 2: Orientation-Dependent Energy Separation

$\begin{array}{cccc}\begin{array}{c}\text { Crystal } \\ \text { orientation }\end{array} & \begin{array}{c}\text { Angle w.r.t z- } \\ \text { axis }\end{array} & \text { C-HH(eV) } & \text { HH-LH(eV) } \\ (100) & 0^{\circ} & 1.84 & \\ (110) & 90^{\circ} & 1.80 & 0.09 \\ (111) & 54.7^{\circ} & 1.78 & 0.4 \\ (113) & 25.2^{\circ} & 1.86 & 1.19 \\ (131) & 72.5^{\circ} & 1.87 & 0.78 \\ & & & 0.98\end{array}$

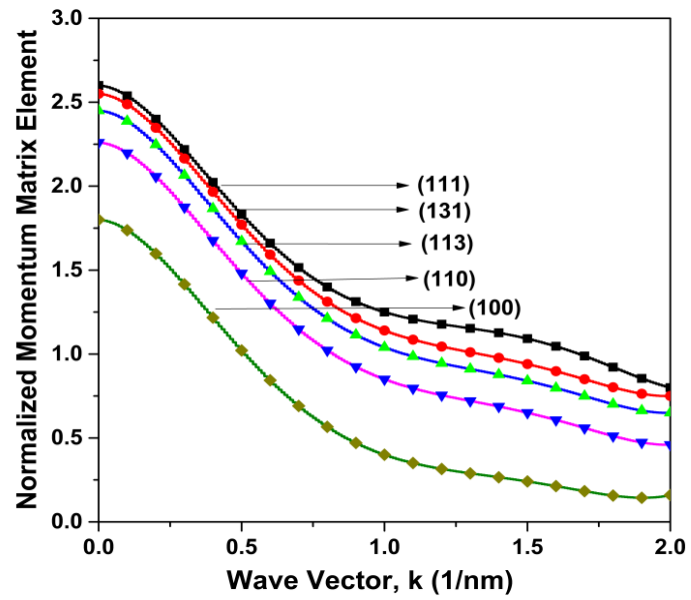

Fig 3: Crystal orientation-dependent normalized momentum matrix element squared for C-HH transition

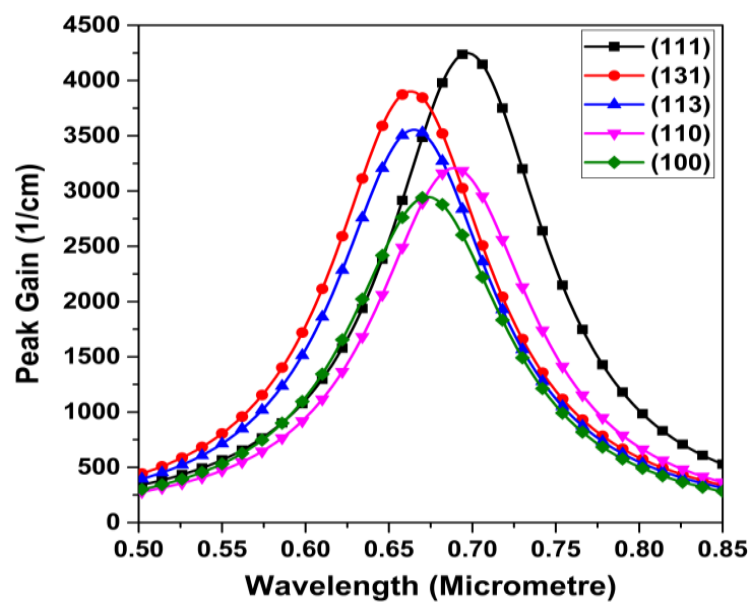

Fig 4: Crystal orientation-dependent Optical Emission Profile 


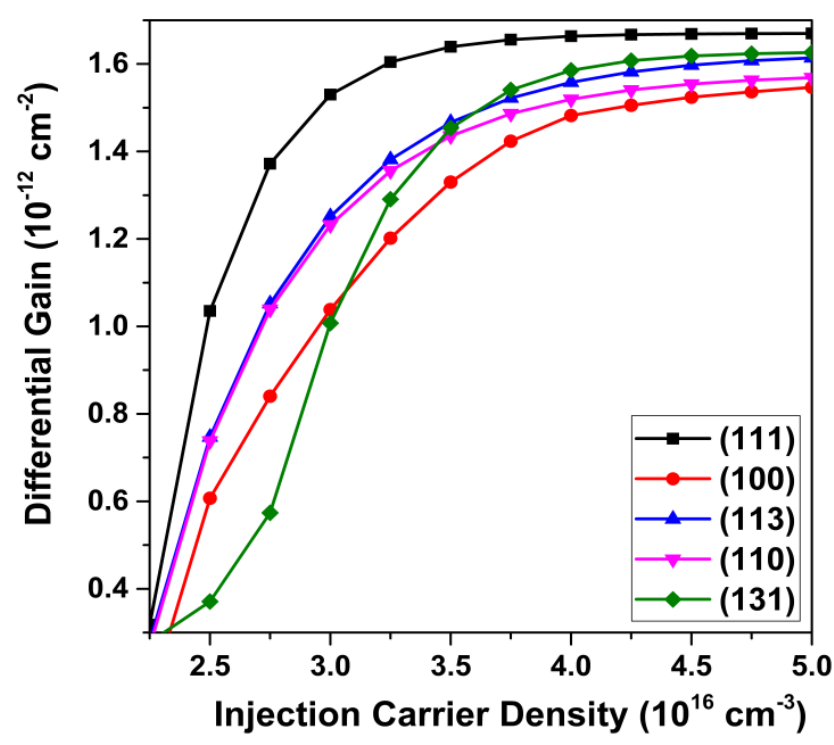

Fig 5: Differential gain in Different Orientation

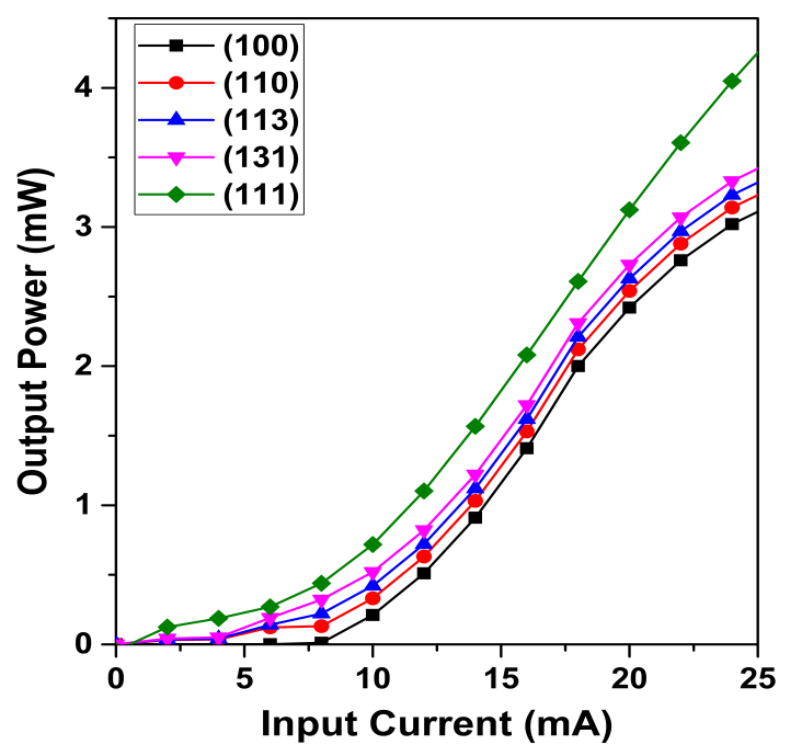

Fig 6: Orientation-dependent Output Power Characteristics

\section{CONCLUSION}

$\mathrm{AlGaAs}$ laser emitting around $670 \mathrm{~nm}$ is analysed in (100) as well as non-conventional crystal orientation in MATLAB and PSPICE environment using 8-band k.p matrix and subsequent tensor rotation formula. It's seen that crystal orientation alters energy gap between conduction and valence sub-bands. Highest momentum of carrier transition between conduction and heavy hole band and peak gain is obtained in (111) crystal orientation (an angle of $54.7^{\circ}$ w.r.t (100) plane) because of highest energy separation between valence subbands in this orientation. It is also seen that peak emission wavelength of the proposed laser shows adjustable characteristics and it varies from 670 to $695 \mathrm{~nm}$ for changing crystal orientation from (131) to (111). Alongside with this, from the inspection of output optical power and threshold current, it can be said that (111)-oriented structure gives the best performance. As modern era's lightwave communication technology is now tending towards over $40 \mathrm{gbit} / \mathrm{s}$ class architecture, (111)- oriented $\mathrm{AlGaAs}$ vertical cavity surface emitting laser system is a prospective candidate for ultra-high speed ICs.

\section{REFERENCES}

[1] S. F. Yu, "Analysis and Design of Vertical Cavity Surface Emitting Lasers", Wiley series in lasers and applications, 2003.

[2] H. Tanobe; F. Koyama; K. Iga, "Spectral linewidth of $\mathrm{AlGaAs} / \mathrm{GaAs}$ surface-emitting laser", Electronics Letters (Volume: 25, Issue: 21, 12 Oct. 1989)

[3] S.-J. Yih; S.-C Lee, "AlGaAs/GaAs surface emitting laser diode with curved reflector", Electronics Letters (Volume: 26, Issue: 18, 30 Aug. 1990)

[4] William Posten et al., "Low-Level Laser Therapy for Wound Healing: Mechanism and Efficacy", Wiley, 2005 
[5] A. Schindl et al., "Direct stimulatory effect of lowintensity $670 \mathrm{~nm}$ laser irradiation on human endothelial cell proliferation", Wiley, 2003

[6] T. Ohtoshi, T. Kuroda, A. Niwa, and S. Tsuji, "Dependence of optical gain on crystal orientation in surface-emitting lasers with strained quantum wells" , Applied Physics Letters 65, 1886 (1994)

[7] A. Niwa, T. Ohtoshi and T. Kuroda, "Orientation dependence of optical properties in long wavelength strained quantum-well lasers, IEEE J. Selected Top. Quantum Electron. 1, pp. 211- 217 (1991)

[8] Bakin et al.," Simulation of the orientation-dependent growth of InGaAs/InP by MOVPE, Journal of Applied Physics 76, 1994

[9] G. Zwinge, H.H. Wehmann, A. Schlachetzki, and C. C. Hsu, "Orientation-dependent growth of InGaAs/InP for applications in laser diode arrays", Journal of Applied Physics 74, 5516 (1993)

[10] M.M.Hasan, M.R.Islam, K. Teramoto, "Crystallographic orientation-dependent optical properties of GaInSb midinfrared quantum well laser", Optik - Int. J. Light Electron Opt., Elsevier (2011)
[11] Yae Okuno et. al., "Direct Wafer Bonding of III-V Compound Semiconductors for Free-Material and FreeOrientation Integration", IEEE Journal of quantum electronics, vol. 33, no. 6, June 1997

[12] M El Allali et al, "Experimental determination of the GaAs and $\mathrm{Al}_{\mathrm{x}} \mathrm{Ga}_{1-\mathrm{x}} \mathrm{As}$ band-gap energy dependence on temperature and aluminum mole fraction in the direct band-gap region", Phys. Rev. B 48 (1993)

[13] Craig Pryor, Eight-band calculations of strained InAs/GaAs quantum dots compared with one, four, and six-band approximations, Physical Review B, 03/1998

[14] S.L.Chuang, "Physics of Optoelectronic Devices", Wiley, 1995

[15] Pablo V. Mena and Thomas A. DeTemple, "RateEquation-Based Laser Models with a Single Solution Regime", Journal of Lightwave Technology, vol. 15, no. 4, April 1997

[16] Giammarco Rossi, Roberto Paoletti, "SPICE Simulation for Analysis and Design of Fast 1.55 m MQW Laser Diodes", Journal of Lightwave Technology, vol. 16, no. 8, August 1998

[17] http://www.ioffe.ru/SVA/NSM/Semicond/AlGaAs/bands tr.html 